\section{Construcción de ciudadanía y \\ Educación al Desarrollo. Fronteras y horizontes de una experiencia local}

\author{
María Angélica Sabatier \\ Directora Ejecutiva del Programa \\ de Formulación de Proyectos Sociales. \\ Secretaría de Desarrollo Social. \\ Municipalidad de Santa Fe. \\ Eduardo Gallo \\ Coordinador Ejecutivo del Programa \\ Mejoramiento Integral del Oeste. \\ Santa Fe Hábitat: agencia para \\ el desarrollo social y urbano. \\ Municipalidad de Santa Fe.
}

Construir ciudadanía /

Intervenciones

\author{
Magalí Dolzani \\ Integrante Junior del Equipo Local \\ del Proyecto. Estudiante avanzada \\ de Ciencias Políticas. Universidad \\ Nacional de Rosario. \\ Laura Cerioni \\ Integrante Junior del Equipo Local \\ del Proyecto. Estudiante avanzada \\ de Licenciatura en Comunicación \\ Social. Universidad Nacional \\ de Entre Ríos.
}

\title{
Introducción
}

"Construir un Desarrollo Posible" ha sido una intervención interinstitucional gestada en el marco de la cooperación al desarrollo realizada con recursos del Programa EUROPaid, coordinada por el Gobierno de la Regione Marche (Italia), y llevada a cabo por organizaciones no gubernamentales europeas, la Universidad de Maceratta (Italia), la Municipalidad de la Ciudad de Santa $\mathrm{Fe}$ —donde se llevó adelante la experiencia de la que aquí se da cuenta - y el gobierno de la provincia del mismo nombre, que conformaron un diversificado conjunto de socios. ${ }^{1}$

Por su estructura, el proyecto —apoyado en los Objetivos del Milenio y a partir de un objetivo central común ${ }^{2}{ }^{2}$ desplegó acciones con propósitos particulares en cada territorio y posibilitó el contacto con las mejores experiencias italianas y europeas en redes de Educación al Desarrollo, teniendo en cuenta —al mismo tiempo- fundamentalmente la visión local sobre las relaciones entre construcción de ciudadanía, Educación al Desarrollo y fortalecimiento de la sociedad civil.

En el caso de Santa Fe Ciudad, los objetivos particulares aludieron específicamente a dar sustento a una comunidad capaz de afrontar los desafíos de su tiempo y al potenciamiento de las capacidades de proyectación y programación de los actores locales, operando en red. Ejecutada a partir de mayo de 2010 y por el término de 18 meses, la intervención tuvo su punto de partida en el abordaje de los conceptos estructurantes así como de las relaciones y nexos operativos entre ellos. Articulada y coordinada por la Municipalidad,

1) Proyecto EUROPaid /126341. Socios: ABRAÇO (ONG, Portugal); CESTAS, Centro di Educazione Sanitaria e Tecnologie Appropriate Sanitarie (ONG, Italia); CVM, Centro Volontari Marchigiani (ONG, Italia); Circolo Culturale Africa (ONG, Italia) CISP, Comitato Internazionale per lo Sviluppo dei Popoli (ONG, Italia);
CISV, Comunità Impegno Servizio Volontariato (ONG, Italia); COSPE (ONG, Italia); Mondo Solidale (Cooperativa, Italia); Solidarités Jeunesses (ONG, Francia); S.O.S. Missionario (ONG, Italia); Università degli Studi di Macerata (Italia). Integrantes latinoamericanos: e Gobierno de la Provincia de Santa Fe y siguió un modelo de cogestión entre actores gubernamentales y no gubernamentales, los cuales participaron de modo continuo en la identificación y ejecución de las actividades al tiempo que se iba consolidando el espacio de producción a través de un Grupo de Trabajo Ampliado.

Desde esta perspectiva, y tomando a la Educación al Desarrollo como herramienta efectiva, se relevaron intereses, necesidades y sobre todo se pensaron las acciones orientadas a fortalecer la condición ciudadana individual y colectiva.

\section{Las cuestiones conceptuales principales:} construir ciudadanía, educación y comunicación al desarrollo La acción local abre el juego a todos los actores implicados a través de un Coloquio Introductorio, ${ }^{3}$ con eje principal en el debate de ideas ${ }^{4}$ en torno a: la participación ciudadana en espacios locales; las interacciones entre gobierno y sociedad civil; la intervención de la sociedad en las políticas públicas; la descentralización entendida como la transferencia de poder a entidades estatales locales, ya que esto facilita la relación gobierno-ciudadanos e incrementa la eficiencia y la democratización de los espacios de decisión y es un mecanismo clave del desarrollo territorial que opera en un escenario local de interacción, esto es, la trama local de actores, relativamente autónoma y comprometida con el desarrollo de su territorio; la la Municipalidad de la Ciudad de Santa Fe de la Vera Cruz.

2) El objetivo principal: "Contribuir a un sistema regional de Educación al Desarrollo para la Cooperación Descentralizada"; subsidiariamente: "Propender a la creación de redes sustentables de solidaridad y cooperación local, regional e internacional".
3) "Construir un desarrollo posible. Apuntes de trabajo y síntesis de resultados", mayo 2012, p. 21. 4) "La participación ciudadana en espacios locales", conferencia de apertura del Coloquio Introductorio, a cargo de la Dra. Adriana Rofman de la Universidad Nacional de Gral. Sarmiento. 
democracia de alta intensidad, ${ }^{5}$ es decir, más democracia y eficacia en el gobierno y gestión en red, multiplicidad de mecanismos y centros de decisión, cooperación no jerárquica.

Todas estas nociones tienen que ver con el empoderamiento ${ }^{6}$ de los actores sociales individuales y colectivos, con la

co-construcción de condiciones ciudadanas potentes, responsables y participativas, que interpelan no sólo a las prácticas políticas sino esencialmente a las prácticas formativas formales y no formales, entre las que la transferencia del conocimiento socialmente apropiable adquiere particular relevancia en clave de desarrollo humano.

Para reflexionar sobre esto, se tomó como base a Manfred MaxNeef, quien expresa que:

"Necesidades humanas, autodependencia y articulaciones orgánicas son los pilares fundamentales que sustentan el desarrollo a escala humana. Pero para servir a su propósito sustentador deben, a su vez, apoyarse sobre una base sólida. Esa base se construye a partir del protagonismo real de las personas, como consecuencia de privilegiar tanto la diversidad como la autonomía de espacios en que el protagonismo sea realmente posible. Lograr la transformación de la persona —objeto en persona- sujeto del desarrollo es, entre otras cosas, un problema de escala; porque no hay protagonismo posible en sistemas gigantísticos organizados jerárquicamente desde arriba hacia abajo". (Max-Neef, 1993:30)

El desarrollo a escala humana apunta —entonces— hacia una necesaria profundización democrática y una concepción "desde abajo hacia arriba" (botton up) de las estrategias sociales. En este punto corresponde mencionar que la expuesta es la concepción vigente de la Cooperación Descentralizada al Desarrollo, es la realizada por administraciones subestatales y está caracterizada por la descentralización de iniciativas y la incorporación de actores de la sociedad civil. Este enfoque de cooperación reinterpreta el rol de las ONG como intermediarias ante el surgimiento de un nuevo espacio para los agentes locales al tener éstos mayor protagonismo y responsabilidad en la concepción de las propuestas de desarrollo económico y social. Tiene en la Educación al Desarrollo (EaD) una componente de alta significación y sus palabras clave son: pluralismo institucional, desarrollo participativo y descentralización.

Todo esto implica -entre otras cuestiones- la construcción sostenida de una ciudadanía diferente como meta y la adopción de la Educación al Desarrollo como herramienta estratégica. En su Diccionario para la Educación al Desarrollo, Hegoa ${ }^{7}$ la concibe como un proceso educativo encaminado a generar conciencia crítica, a promover una ciudadanía global políticamente activa y comprometida y a facilitar herramientas para la participación y la transformación social respecto de justicia y solidaridad. Desde el Diccionario de Acción Humanitaria y Cooperación al Desarrollo, ${ }^{8}$ se considera a la EaD como un proceso dinámico, interactivo y participativo orientado a la formación integral de las personas. Es un proceso a medio o largo plazo que posibilita la capacitación, formación y puesta en marcha de estrategias de transformación de la realidad global por parte de personas o grupos que la componen, por lo que impregna e influye sobre cuatro dimensiones diferentes: la personal, la de la comunidad local, la nacional y la internacional. Es un concepto dinámico que va evolucionando con las personas con las que interactúa, en el marco de la educación bien sea formal, no formal o informal. ${ }^{9}$

En orden a un fortalecimiento del capital humano y social, hacer EaD incluye -entre otras- acciones que propenden a: asegurar la equidad de género; incrementar la conciencia ambiental y el involucramiento por la sostenibilidad; promover políticas y acciones de prevención en salud y calidad de vida; sensibilizar en materia de derechos humanos (derechos sociales, derechos políticos, derechos económicos, derechos civiles y derechos culturales); fomentar la participación ciudadana; orientar actividades de
5) En su libro Democracia de alta intensidad. Apuntes para democratizar la democracia, Boaventura de Sousa Santos hace hincapié en que no hay una sino varias concepciones de democracia y propone la democracia participativa como alternativa a la democracia representativa. Concibe a la democracia participativa como una sustitución de las relaciones de poder por relaciones de autoridad compartida.

6) Clara Murguialday, Karlos Pérez de Armiño y Marlen Eizaguirre entienden al empoderamiento como un proceso por el cual las personas fortalecen sus capacidades, confianza, visión y protagonismo como grupo social para impulsar cambios positivos de las situaciones que viven. La filosofía del empoderamiento tiene su origen en el enfoque de la Educación Popular desarrollada a partir del trabajo de Paulo Freyre en los años 60, y ambas están muy ligadas a los denominados enfoques participativos, presentes en el campo del desarrollo desde los 70 . 7) Hegoa, organización sin ánimo de lucro del País Vasco, que trabaja en la promoción del desarrollo humano, amplía estos conceptos y establece que la EaD pretende construir una ciudadanía global cuyas demandas, necesidades, preocupaciones y análisis se tengan en cuenta a la hora de la toma de decisiones políticas, económicas y sociales. La entiende como una educación que busca que las personas se comporten como actores capaces de realizar análisis críticos de la realidad y de imaginar y proponer modelos de desarrollo satisfactorios, sustentables y equitativos.

8) En este diccionario, Marlen Eizaguirre reconoce que existen dos grandes interpretaciones de la EaD. Por un lado, una concepción específica, que limita sus contenidos a los problemas norte-sur; por el otro, una concepción más amplia e integral según la cual la EaD sería una denominación genérica que englobaría otros tipos de educación: intercultural, en valores, para la solidaridad, para la tolerancia, para la paz, etcétera.

9) En lo formal, promueve valores y actitudes referidos a justicia-equidad, tolerancia, solidaridad y cooperación, entre otros. No es un contenido sino un posicionamiento ante la enseñanza que pretende suscitar un cambio positivo de actitudes y prácticas a través de una concepción multidireccional del proceso de enseñanza-aprendizaje basado en una construcción dinámica de los saberes. En lo no formal e informal, comprende acciones como campañas de sensibilización, movilizaciones, denuncias, lobby político, etcétera. 
voluntariado social; generar apoyo y adhesión a diferentes proyectos culturales, impulsar el trabajo en red de organizaciones sociales, instituciones públicas y privadas; promover acciones de formación al consumidor y visibilizar la necesidad de la gestión de riesgos. Como algo no escindible de la EaD apareció, por interés de los propios actores, la cuestión de la Comunicación al Desarrollo en tanto instrumento que contribuye a lograr los objetivos de aquélla y la sostenibilidad de los resultados de cada acción, ${ }^{10}$ ya que concibe al sujeto como protagonista activo situado desde donde se enuncia la palabra y no como simple receptor de un mensaje. Así, Educación y Comunicación (EyCaD) constituyen herramientas estratégicas convergentes para el desarrollo a escala humana y por lo tanto estructuran y potencian la construcción de ciudadanía. Conceptualizadas en conjunto, buscan promover una cultura de solidaridad recíproca; y como educativo-comunicativo participativo, uno de los objetivos principales es que los diversos actores aprendan de la experiencia común.

\section{Modalidad de abordaje}

Desde la plataforma de ideas hasta aquí expresada, la intervención se desarrolló como un proceso cooperativo entre pares - sin arriba, sin abajo- a través del cual fluyó la construcción de saberes, la circulación de experiencias, aparecieron temores, expectativas, necesidades, fortalezas, debilidades y, sobre todo, se configuró un espacio en el que fue posible tomar registro de cada una de las cuestiones que emergieron.

La acción local consistió en el abordaje analítico de la EyCaD en la ciudad a través de seminarios con instituciones de la sociedad civil y organismos públicos y privados; el relevamiento de las características de las Organizaciones de la Sociedad Civil (OSC) locales vinculadas a la temática; la documentación de sus relatos $y$, a partir de ello, la inclusión sistemática los encuentros de trabajo e instancias colectivas de intercambio con otros actores institucionales tanto públicos como privados.

Se siguió lo que se conoce como "estrategia caracol", partiendo de un pequeño núcleo de representantes de instituciones que por su experiencia, trayectoria y conocimiento del campo conceptual y de trabajo, posibilitaron la incorporación gradual y sistemática de otros actores - principalmente OSC_, vinculados de un modo u otro a la problemática de la EyCaD.

De este modo, ya en el inicio de la acción local, se conformó el Grupo de Trabajo Ampliado (GTA), integrado por los miembros del
Equipo Local de Proyecto -ELP-, ${ }^{11}$ representantes de OSC, ${ }^{12}$ las ONG Italianas con asiento en la ciudad, CISP y GVC, distintas áreas del gobierno local ligadas al desarrollo social y la Universidad Nacional del Litoral a través de la Secretaría de Extensión. Actor significativo del desarrollo local, la UNL, a través de la función de extensión, ha venido realizando en las últimas décadas un fuerte trabajo territorial basado en el relacionamiento con los actores sociales, centrado en la transferencia del conocimiento socialmente apropiable y realizado a la manera en que se concibe la EaD. Hasta fines del año 2007 no había habido por parte del gobierno local un acompañamiento consistente de esa trayectoria. Luego, en coincidencia con el enfoque extensionista y su modalidad participativa, el municipio, con el desembarco de "Construir un Desarrollo Posible", estrechó una alianza natural con el objeto de profundizar la construcción de trayectorias a recorrer en distintos sentidos por los actores sociales en la búsqueda de mejores condiciones para encarar sistemáticamente la transformación de la realidad.

\section{Acerca del recorrido efectuado}

El trabajo desarrollado excedió las expectativas iniciales; la dinámica alcanzada hizo necesario profundizar las acciones de formación y fortalecimiento e hizo posible sentar las bases de una red local. Al relevamiento de $45 \mathrm{OSC}$ - cuyos resultados constan en páginas 26 a 37 de la publicación reseñada en la nota 3- se sumaron instancias de fortalecimiento cuya necesidad emergió como resultado de la interacción permanente.

El Seminario "La Educación al Desarrollo en Santa Fe, un camino firme hacia la construcción de ciudadanía"13 puso de manifiesto la necesidad de repensar lo educativo desde los más tempranos años de vida, rompiendo el molde de lo vigente, y desde un enfoque de ciudadanía democrática como narrativa para la EaD.

El taller denominado "Educación al desarrollo en el contexto de redes locales, regionales e internacionales de cooperación" hizo factible la identificación de los 5 principios básicos — desde la mirada local- de la EyCaD: funcionamiento en red; consenso en el entramado político; cooperación interinstitucional e interregional; igualdad y cultura de la solidaridad recíproca. Coordinado por la Secretaría de Extensión de la UNL, permitió a los participantes establecer las estrategias necesarias para sostener los principios mencionados en las acciones locales: el intercambio de información y experiencias para organizar proyectos concretos; la conformación de redes con fines específicos; la promoción de acciones que
10) Acción, es entendida - para la cooperación al desarrollo- como un conjunto capaz de configurar un proyecto de desarrollo.

11) El Equipo Local de Proyecto estuvo integrado por los autores del presente artículo.
12) Integraron el Grupo de Trabajo Ampliado las OSCs Juanito Laguna, Madres Inundadas, Cooperativa Santa Rosa Avanza, Centro Interdisciplinario para la Prevención de Enfermedades de Transmisión Sexual y SIDA (CIPRESS), Lugar Barrial de Ajedrez (LBA),
Asociación Civil Suzy Tomas, Quo Vadis, Palabras, Servicio de Educación Popular (SEP), Comitato Internazionale per lo Sviluppo dei Popoli (CISP), Gruppo di Volontariato Civile (GVC) y el periodista Aldo Quiroz, en su condición de referente de medios de comunicación comunitarios. 13) Realizado en abril de 2011 en Rosario, por convocatoria de la Subsecretaría de Cooperación y Relaciones Internacionales del Gobierno de la Provincia de Santa Fe en calidad de socio del Proyecto. 
impulsen nuevas pautas culturales, que cambien la lógica de lo individual por la lógica del tejido en red; el fortalecimiento de los espacios públicos; la promoción del encuentro entre vecinos de diferentes sectores; la validación de las propuestas por parte de los beneficiarios de los resultados para lograr su interés, involucramiento y participación activa.

EI CISP, en su condición de socio, y el Área de Cooperación Internacional del Centro Balear compartieron su experiencia sobre gestión de proyectos con financiamiento vía la cooperación descentralizada, uno como ONG y el otro como expresión local de un gobierno subnacional.

Con el propósito de reforzar la formación de recursos humanos en torno a la formulación de proyectos de cooperación - significativa demanda expresada por las OSC— se presentaron dos candidaturas a la 5ta. Convocatoria para la Formulación de Iniciativas en el Campo de la Inclusión Social que realiza periódicamente Innova Integra-Mercociudades.

En el mismo sentido se llevaron a cabo las Jornadas Introductorias al Ciclo de Gestión de Proyectos de Cooperación, consistentes en dos encuentros intensivos a cargo del Equipo Local de Proyecto.

En éstas se abordaron cuestiones como la construcción de consensos en torno al diagnóstico del problema, la producción de la información necesaria para el análisis y comprensión de sus causas, la cooperación para la delimitación de la acción capaz de contribuir a la modificación favorable de la situación no deseada, la celebración de alianzas y su sostenimiento en todas las etapas y fases del ciclo. En el marco del XI Congreso Iberoamericano de Extensión Universitaria $^{14}$ se comunicaron, en la Mesa de Trabajo "Gestión Mixta de Problemáticas Socio Comunitarias", los resultados alcanzados hasta ese momento.

\section{Hallazgos de esta primera fase}

El trabajo desarrollado deja como enseñanza principal que, si bien distintos actores de la comunidad local vienen desarrollando acciones que se inscriben en el campo de la EyCaD y, por lo tanto de la construcción de ciudadanía, no ha habido una apropiación operativa de estos conceptos como tales. Por ello, los mismos actores han señalado como fundamental que se puedan consolidar estas nociones a través de espacios de debate, de diálogo y producción que impacten sobre el conjunto de la sociedad. Resulta muy oportuno señalar que las principales convergencias y coincidencias se presentaron en cuanto a la necesidad de:

14) Organizado por la UNL y realizado en Santa Fe de la Vera Cruz entre el 22 y el 25 de noviembre de 2011 bajo el lema "Integración, Extensión, Docencia e Investigación para la Inclusión y Cohesión Social". construir una estrategia interinstitucional común y a largo plazo de EyCaD, coordinar acciones de manera conjunta entre el gobierno local, la sociedad civil, las universidades y otras instituciones; impulsar conferencias, foros, debates, talleres, y sobre todo experiencias concretas y durables; definir prioridades de educación y comunicación al desarrollo para la ciudad; capacitar formadores sobre el uso de metodologías activas y participativas; alentar a las instituciones educativas para que incorporen el enfoque en el proyecto institucional y la currícula escolar; realizar campañas de sensibilización del conjunto de la sociedad y promover valores como solidaridad y cooperación; impulsar la inclusión del enfoque en la educación de los niños desde su más temprana edad con integración de la familia, y fomentar acciones solidarias de modo sistemático. Resultados éstos de una instancia que los actores entienden como el inicio de una larga trayectoria, son concebidos por ellos como los puntos de partida de una fase futura.

\section{Bibliografía}

Argibay, Miguel; Celorio, Gema y Celorio, Juanjo (1997). "Cuadernos de Trabajo de Hegoa. Educación para el Desarrollo. El espacio olvidado de la cooperación.", no 19. Disponible en: http://fongdcam.org/manuales/educaciondesarrollo/datos/ docs/A_docs/b_1_3_espacio_hegoa.pdf - Agosto 1997.

Argibay, Miguel y Celorio, Gema (2005). "La Educación para el Desarrollo". Manuales de Formación. Cooperación Pública Vasca. Disponible en: http://biblioteca. hegoa.ehu.es/system/ebooks/15211/original/La_educacion_para_el_desarrollo.pdf Caballero González, Iztiar (coord.) (2004). "Cuaderno de Trabajo de Educación para el Desarrollo". Edición UNESCO Extea. Disponible en: http://educaciondesarrollo. fongdcam.org/files/2012/01/caballero.pdf

Celorio, Gema y López de Munain, Alicia (coords.). Diccionario de Educación para el Desarro/lo. Hegoa. Disponible en: http://pdf2.hegoa.efaber.net/entry/content/158/ diccionario_2.pdf

De Sousa Santos, Boaventura (2004). "Democracia de Alta Intensidad. Apuntes para democratizar la democracia.” La Paz: Edit. Unidad de Análisis e Investigación del Área de Educación Ciudadana de la Corte Nacional Electoral de la República de Bolivia. $1^{\text {a }}$ edición.

Max-Neef, Manfred (1993). "Desarrollo a escala humana." Montevideo: NordanComunidad.

Municipalidad de Santa Fe de la Vera Cruz (2012). "Construir un desarrollo posible: Apuntes de trabajo y síntesis de resultados." Editor Responsable María Angélica Sabatier, ISBN en trámite.

Pérez de Armiño, Karlos (dir.). Diccionario de Acción Comunitaria y Cooperación al Desarrollo. Icaria y Hegoa Editores, Diccionario online: http://www.dicc.hegoa.ehu.es/ 